\title{
Seroprevalence of Toxoplasma gondii infection amongst residents of Tanga District in north-east Tanzania
}

\author{
E.S. SWAI ${ }^{*}$ and L.SCHOONMAN ${ }^{2}$ \\ ${ }^{1}$ Veterinary Investigation Centre, P.O. Box 1068, Arusha, Tanzania \\ ${ }^{2}$ Tanga Dairy Trust, P.O. Box 1720, Tanga, Tanzania.
}

\begin{abstract}
Toxoplasmosis is a zoonotic disease, recognized as a serious public health problem worldwide. Toxoplasma gondii infection has become a major public health concern in recent years due to the ravaging HIV / AIDS pandemic. A serological survey was carried out in Tanga district of north-eastern Tanzania to assess $T$. gondii infection rates among occupationally-exposed groups including abattoir workers, livestock keepers, animal health workers and other groups. The survey was undertaken in November 2005 using modified Eiken latex agglutination test (LAT). Antibodies to T. gondii were detected in $91(46 \%)$ of the 199 individuals studied. T. gondii seroprevalence was slightly higher amongst males $(46.2 \%)$ than females $(43.3 \%)$ although the difference was not significant $(P>0.05)$. Individuals, $\leq 20$ and $\geq 60$ years old had the highest prevalence of $60 \%$ and $61.5 \%$, respectively. The lowest prevalence of $(35.7 \%)$ was observed amongst the 50-60 years age group. The seroprevalence of toxoplasma antibodies was significantly higher amongst individuals who keep livestock $(52.2 \%)$ and abattoir workers $(46.3 \%)$. These results suggest exposure to T. gondii infection is present among residents of Tanga district in Tanzania and strengthen further on previous findings that consumption of raw or undercooked meat and keeping pets especially cats presents more of the risk factors than occupational groups. It also emphasizes on the necessity to create awareness of this disease, and advocate protection of risky groups from exposure to infected meat and contaminated environment.
\end{abstract}

Key words: occupational group, seroprevalence, Toxoplasma gondii, Tanzania

\section{Introduction}

Toxoplasmosis, a zoonotic disease of world wide distribution, is an infection caused by an apicomplexan single-celled, obligate intracellular protozoan parasite called Toxoplasma gondii (Ryan \& Ray, 2004; Klevar, 2007). World wide, around one third of human population is estimated to carry a Toxoplasma infection (Montoya \& Liesenfeld, 2004). The infection is most commonly acquired from contact with cats and their faeces or by ingesting tissue cysts in under-cooked or uncooked meat (Schlundt et al., 2004). Infected persons develop either mild flu-like illness characterised by fever, body aches, headaches and sore throat or no illness at all. People with a weakened immune system, such as those infected with HIV or pregnant women, may become seriously ill, and infection can occasionally be fatal (Lindstrom et al., 2006; Negash et al., 2008). The parasite can cause encephalitis and neurologic diseases and can affect the heart, liver and eyes.

In Tanzania, as in most countries of sub-Saharan Africa, agriculture, dominated by crop-livestock production systems, is the main dependable source of employment, household economy and livelihood. Under such situation, exposure of human beings to animals is quite high. Livestock keepers, veterinarians/animal health personnel, abattoir workers and the livestock products consumers are particularly at risk. The disease is known to occur in Tanzania (Gill \& Mtimavalye 1982; Gille et al., 1992; Doehring et al., 1995), however, there is no published literature available on the toxoplasmosis status in these occupationallyexposed groups. Establishing disease status, particularly in these high risk exposed groups is an important component in the assessment of levels of past or present exposure to this disease, a crucial input that will enable proper interventions to be undertaken.

In this paper we present the results of a cross sectional serological study conducted in apparently healthy occupationally-exposed inhabitants of Tanga, Tanzania. The aim was to examine, identify and quantify the relative importance of different occupations that may determine the likelihood of human exposure to

* Correspondence: Dr. E.S. Swai; E-mail: ESwai@gmail.com 
infection by $T$. gondii. The information generated will guide development of appropriate disease control interventions.

\section{Materials and Methods}

\section{Study area and population}

This cross-sectional study was conducted in Tanga district in north-east Tanzania (Latitude $4^{\circ} 21^{\prime}-6^{\circ} 14^{\prime} \mathrm{S}$; Longitude $\left.36^{\circ} 11^{\prime}-38^{\circ} 26^{\prime} \mathrm{E}\right)$. The district has a human population of around 242,640 inhabitants growing at $2.9 \%$ per annum (URT, 2002). Most rural inhabitants are subsistence farmers, whilst a number of residents of Tanga City are business men/ women or work in industries. Tanga district has a hot and humid tropical climate with two rainy seasons: long rains in March-May and short rains in November-December. The mean annual rainfall varies from 500 to $1400 \mathrm{~mm}$. The relative humidity of the day ranges from $60 \%$ to $90 \%$ for most of the year. Monthly mean temperatures range from $15^{\circ} \mathrm{C}-35^{\circ} \mathrm{C}$. Subjects for the study were selected according to the willingness of the individual to be included in the study frame, for testing.

\section{Data collection}

A pre-tested individual Kiswahili questionnaire with closed ended questions was administered face-to-face to all study participants. Information sought included demographic (age, gender, location) and occupation (categorised as abattoir workers; livestock keepers; nonlivestock keepers; animal health workers) and others. The 'others' category comprised people from the general community. Other information collected included contact with other livestock such as cat at home, type of activities engaged with, as well as awareness regarding zonooses including toxoplasmosis. The study was conducted during November, 2005.

\section{Ethical considerations}

During survey visits, interviewers introduced themselves and explained the objectives and all procedures to all potential interviewees. A written consent form was obtained from adults or guardians of those individuals aged less than 18 years prior to inclusion. Ethical approval was granted by the Medical Research Coordinating Committee of the National Institute for Medical Research. Research clearance was obtained from the Tanzania Commission for Science and Technology.

\section{Laboratory analysis of serum samples}

A venous blood sample was obtained from each participant. Immediately after puncture, blood samples were stored at $4-8^{\circ} \mathrm{C}$ and forwarded on the same day to the laboratory of Tanga Regional Hospital, which was responsible for all serologic testing. Testing was performed using the Eiken latex agglutination test. Briefly, samples were diluted in AMP buffer, $0.2 \mathrm{M}$ 2-amino-2-methyl1-propanol-HCl (Sigma Ltd, UK). The samples were tested in only two screening dilutions, 1:16 and 1:32. A positive control serum was tested alongside the samples. The test was performed according to the manufacturer's instructions, with the only modification that, V-well microtitre plates were used instead of U-well. The test was read following overnight incubation at room temperature. Antibody titres of 1:32 were considered as positive and titres of 1:16 weak positive. A titre of 1: 32 or greater was taken as an index of seropositivity in this study.

\section{Data analysis}

Questionnaire and laboratory data were handled and analysed using Epi-info (version 6.04d) (CDC, Atlanta, USA). The differences in $T$. gondii antibody prevalence were compared across the investigated variables using the Mantel-Haenszel chi-square test. A value of $P<0.05$ was considered significant.

\section{Results}

\section{Descriptive statistics}

A total of 199 sera from women and men were collected. The average age of study participants was 36.6 years (range 14 to 84 ). Most of the sampled subjects $(n=162 ; 81 \%)$ were found in the age group 20-50 years old (Table 1). Two thirds of the sampled subjects were male and one third female. The abattoir group consisted of only males. All of the non-livestock farmers group and almost all persons of the group 'others' did not have cattle at home. Nonlivestock farmers were those involved in other agricultural activities such as crop production. Nine $(23 \%)$ of the abattoir workers and most of the veterinary staff $(\mathrm{N}=10 ; 90 \%)$ reported keeping livestock at home and being involved in cattle keeping related activities. None of the study participants perceived toxoplasmosis to be a zoonotic diseases. 
Table 1: The seroprevalence of $T$. gondii antibodies among individuals in each category investigated during the study $(\mathrm{N}=199)$

\begin{tabular}{|c|c|c|c|c|c|}
\hline Variable & Categories & $\begin{array}{l}\text { No } \\
\text { examined }\end{array}$ & $\%$ & $\begin{array}{l}\text { No } \\
\text { positive }\end{array}$ & $\begin{array}{l}\text { Prevalence, } \\
( \pm 95 \% \text { CIs })\end{array}$ \\
\hline \multicolumn{6}{|c|}{ Occupation group } \\
\hline & Abattoir & 41 & 20.6 & 19 & $46.31(30.6-62.6)$ \\
\hline & Livestock farmer & 67 & 33.6 & 35 & $52.23(39.6-64.6)$ \\
\hline & Non-livestock keepers & 38 & 19.1 & 15 & $39.47(24.0-56.6)$ \\
\hline & $\begin{array}{l}\text { Veterinary } / \text { meat } \\
\text { inspectors }\end{array}$ & 11 & 5.5 & 4 & $36.36(10.9-69.2)$ \\
\hline & Others & 42 & 21.1 & 18 & $42.85(27.7-59.0)$ \\
\hline \multicolumn{6}{|l|}{ Sex } \\
\hline & Female & 67 & 33.7 & 29 & $43.28(31.2-55.9)$ \\
\hline & Male & 132 & 66.3 & 62 & $46.90(38.2-55.8)$ \\
\hline \multicolumn{6}{|l|}{ Age (yrs) } \\
\hline & $\leq 20$ & 10 & 5.02 & 6 & $60(26.2-87.8)$ \\
\hline & $21-30$ & 49 & 24.6 & 25 & $51.02(36.3-65.6)$ \\
\hline & $31-40$ & 67 & 33.6 & 26 & $38.80(27.13-51.5)$ \\
\hline & $41-50$ & 46 & 23.1 & 20 & $43.47(28.9-58.9)$ \\
\hline & $51-60$ & 14 & 7.03 & 5 & $35.71(12.7-64.8)$ \\
\hline & $>60$ & 13 & 6.5 & 8 & $61.53(31.6-86.1)$ \\
\hline & Total & 199 & 91 & 45.72 & $(38.6-52.9)$ \\
\hline
\end{tabular}

\section{Serological response to $\mathrm{T}$. gondii}

The overall seroprevalence of $T$. gondii antibodies in the study population was $45.7 \%$ (95\% [Confidence interval], CI: 38.6-52.9). The highest seropositivity of $60 \%$ and $61.5 \%$ was observed among individual of $\leq 20$ years and $>60$ years respectively, while the lowest $(35.7 \%)$ was amongst the 51-60 years age group although this was not statistically different $(P=0.452)$. No significant difference was observed in the seroprevalence between female $(43.28 \%)$ and male $(46.9 \%)$ participants, neither was observed between different occupational groups $(P=$ 0.621) (Table 1).

\section{Discussion}

The result of this study demonstrated a wide evidence of human exposure to T. gondii in Tanga and is the first report from north-east Tanzania. Similar seroprevalence rates have been reported by Gill \& Mtimavalye (1982) and Doehring et al., (1995). In a study in a rural district in eastern Tanzania, Gille et al.,(1992) reported a prevalence of $4 \%$ among local communities and $47 \%$ among immigrants from other areas of Tanzania. In another study among pregnant women in Dar es Salaam, Tanzania, a seroprevalence of 35\% was reported (Doehring et al., 1995). A higher prevalence $(72.2 \%)$ has been reported among the Hadzabe in northern Tanzania (Bennett et al., 1970). Our findings support the hypothesis that exposure to or background immunity for T. gondii infection is virtually present within the population of Tanga and probably in other parts of north-eastern Tanzania.

The seroprevalence of toxoplasmosis decreases with age, a result which is in contrast with observations made in Nigeria and Congo (Osiyemi et al., 1985; Candolfi et al., 1993). The higher seroprevalence rates found in age group ( $\leq 20$ years) as compared to age group (51-60 years) may be due to an increased exposure of this age group with pet animals (such as cats), poor sanitary habits and probably low level of hygiene during food preparation as a result of low level of awareness of toxoplasmosis. Surprisingly, and in contrast to other studies, we found no significant difference in the seroprevalence between males and females suggesting that sex does not influence exposure to and or/ immune response to $T$. gondii (Candolfi et al., 1993; Uneke et al., 2007) or community-specific habits or behaviours that 
may predispose to exposure. These findings are inconsistent with previous reports from other researchers (Uneke et al., 2007) which indicate that men are more prone to $T$. gondii infection than women.

Human toxoplasmosis has been well studied elsewhere in Africa. The estimated seroprevalence of $45.7 \%$ in the present study is comparable to many other reports from exposed group in Africa. For instance, seroprevalences of $42.6 \%$ and $52.4 \%$ were reported from slaughter house workers in Djibout and Egypt, respectively (Chantal et al., 1996; Ibrahim et al., 1997). Occupational contacts including butchers, slaughter house workers, milkers, and cow attendants in one state of Jos area, Nigeria revealed 22.6\% were infected (Osiyemi et al., 1985). In contrast, cattle breeders and abattoir personnel from Benin gave $87 \%$ positives (Fayomi et al., 1987) and high-risk groups from city of Pointe-Noire in Congo and Addis Ababa in Ethiopia showed a seroprevalence between $41.9 \%$ and $80 \%$, respectively (Candolfi et al., 1993; Woldemichael et al., 1998). Studies of febrile patients in a large hospital in Doha, Qatar yielded 22.9\% while in Gezira Province, Sudan $41.7 \%$ pregnant women were positive (Abdel-Hameed, 1991). The variability in prevalence rates between reports could be due to diagnostic test used, food handling and preparation hygiene, eating habits, level of natural immunity, level of environment contamination with oocysts and the presence of the primary host, the felid (cats) in some of the farming settings.

Limited knowledge on toxoplasmosis as a zoonotic disease as reflected in this study may be due to the general lack of data on toxoplasmosis and inadequate communication between veterinary and human health care professionals. This low awareness is likely to expose them to increased risk of contracting toxoplasmosis, as they might not take proper precaution. For instance eating insufficiently roasted meat, popularly known as 'nyama choma' is common in many restaurants and bars within the study area. Likewise the habit of consuming raw or undercooked meat, however, is still common practice in some communities in Tanzania (Shirima et al., 2003).

The present study documents the first report evaluating seroprevalence of $T$. gondii infection in risky groups in north-eastern Tanzania. The high seroprevalence of $T$. gondii antibodies in the study population clearly demonstrate the public health and zoonotic significance of toxoplasmosis in the study area. In the current study neither contacts with cattle, nor slaughtering cattle were risk factors for toxoplasmosis. Contaminated food, eating raw and under-cooked meat and contact with contaminated soil are most probably the most important source of infection in the area. Findings of the study underscores the need for further research, active surveillance and institution of appropriate preventive and control measures particularly among risky populations.

\section{Acknowledgements}

We are grateful to the generous cooperation of blood donors, public health staff and laboratory personnel at Tanga Regional Hospital.

Received 12 July 2009

Revised 3 August 2009

Accepted 5 August 2009

\section{References}

Abdel-Hameed, A.A. (1991) Sero-epidemiology of Toxoplasmosis in Gezira, Sudan. Journal of Tropical Medicine and Hygiene 94, 329-32.

Bennett, F.J., Kagan, I.G., Barnicot, N.A., Woodburn, J.C. (1970) Helminth and protozoal parasites of the Hadza of Tanzania. Transactions of the Royal Society of Tropical Medicine and Hygiene 64, 857880.

Candolfi, E., Berg, M. \& Kien, T. (1993) Prevalence of Toxoplasmosis in Pointe -Noire in Congo: study of the sampling of 310 subjects. Bulletin de la Société de pathologie exotique et de ses filiales 86, 358-62.

Chantal, J., Bessiere, M.H., Guenno, B le., Magnava,1 J.F. \& Dorchies, P. (1996) A serological survey of some zoonotic diseases among abattoir personnel in Djibouti. (French) Bulletin de la Societe de Pathologie Exotique 89:5, 353-357.

Doehring, E., Reiter-Owona, I., Bauer, O., Kaisi, M., Hlobil, H., Quade, G. \& Hamudu, N.A. (1995) Toxoplasma gondii antibodies in pregnant women and their newborns in Dar-es-Salaam. American Journal of Tropical Medicine and Hygiene 52, 546-8.

Fayomi, B., Duong, T.H., Karagirwa, A., Marquet, N., Djivoh, C. \& Combescot, C. (1987) Serological study of Toxoplasmosis and echinococcosis in cattle breeders and 
abattoir personnel in Benin. Médecine Tropicale : Revue du Corps de santé colonial 47, 149-51.

Gill, H.S. \& Mtimavalye, L. A. (1982) Prevalence of Toxoplasma antibodies in pregnant African women in Tanzania. African Journal of Medical Science 11, 167-170.

Gille, E., Bjorkman, A., Rooth, I., Ljungstrom, I. \& Linder, E. (1992) Low Seroprevalence of Toxoplasma gondii antibodies in a Tanzanian village. Transactions of the Royal Society of Tropical Medicine and Hygiene 86, 263-265.

Ibrahim, B.B., Salama, M.M.I, Gawish, N.I. \& Haridy, F.M. (1997) Serological and histopathological studies on Toxoplasma gondii among the workers and the slaughtered animals in Tanta Abattoir, Gharbia Governate. Journal of the Egyptian Society of Parasitology 27, 273-278.

Klevar, S. (2007) Tissue cyst forming coccidia; Toxoplasma gondii and Neospora caninum as a cause of disease in farm animals. Acta Veterinaria Scandinavica 49 (Suppl. 1): S1doi:10.1186/1751-0147-49-S1-S1

Lindstrom, I., Kaddu-Mulindwa, D.H., Kirond, F. \& Lindh, J, (2006) Prevalence of latent and reactivated Toxoplasma gondii parasites in HIV-patients from Uganda. Acta Tropica 100, 218-222.

Montoya, J. \& Liesenfeld, O. (2004) Toxoplasmosis. Lancet 363 (9425), 19651976.

Negash, T., Tilahun, G. \& Medhin, G. (2008) Seroprevalence of Toxoplasma gondii in Nazareth town, Ethiopia. East African Journal of Public Health 5, 211-214.

Osiyemi, T.I., Synge, E.M., Agbonlahor, D.E. \& Agbavwe, R. (1985) The prevalence of Toxoplasma gondii antibodies in man in Plateau State and meat animals in Nigeria. Transactions of the Royal Society of Tropical Medicine \& Hygiene 79, 21-3.

Ryan, K.J. \& Ray, C.G. (editors) (2004) Sherris Medical Microbiology (4 ${ }^{\text {th }}$ Edn.). McGraw Hill. pp. 723-727.

Schlundt, J., Toyofuku, H., Jansen, J. \& Herbst, S. A. (2004) Emerging food-borne diseases, Revue Scientifique et Technique, Office International des Épizooties 23, 513-533.

Shirima, G.M., Fitzpatrick, J., Cleaveland, S., Kambarage, D.M., Kazwala, R.R., Kunda, J. \& French, N.P. (2003) Participatory survey on zoonotic diseases affecting livestock keeping communities in Tanzania. Journal of Animal \& Veterinary Advances 2, 253-258.

Uneke, C.J., Duhlinska, D.D., Ngwu, B.A. \& Njoku, M.O. (2007) Seroprevalence of Toxoplasma gondii infection in Kwal, a rural distraction of Plateau-Nigeria. African Journal of Medical Science 36, 109113.

URT (2002) Population and household census. United Republic of Tanzania. Available at: http: // www.tanzania.go.tz/ cencus/table5.htm.2002; Accessed April, 2009.

Woldemichael, T., Fontanet, A.L., Sahlu, T., Gilis, H., Messele, T., Rinke de Wit, T.F., Yeneneh, H., Coutinho, R.A. \& Van Gool, T. (1998) Evaluation of the Eiken latex agglutination test for anti-Toxoplasma antibodies and seroprevalence of Toxoplasma infection among factory workers in Addis Ababa, Ethiopia. Transactions of the Royal Society of Tropical Medicine E Hygiene 92, 401-403. 